\title{
RF AND MAGNETIC MEASUREMENTS ON THE SPARC PHOTOINJECTOR AND SOLENOID AT UCLA
}

\author{
J.B. Rosenzweig, A.M. Cook, M.P. Dunning, P. Frigola, G. Travish, UCLA Dept. of Physics and \\ Astronomy, Los Angeles, CA 90095, C. Sanelli, F. Tazzioli, INFN-LNF, Frascati (RM) 00044, \\ Italy, and D.T. Palmer, SLAC, Menlo Park, CA 90120
}

\section{Abstract}

The rf photocathode gun and the solenoid for the SPARC project at INFN-LNF (Frascati) have been fabricated and undergone initial testing at UCLA. The advanced aspects of the design of these devices are detailed. Final diagnosis of the tuning of the RF gun performance, including operating mode frequency and field balance, is described. The emittance compensating solenoid magnet, which is designed to be tuned in longitudinal position by differential excitation of the coils, has been measured using Hall probe scans for field profiling, and pulsed wire methods to determine the field center. Comparisons between measurements and the predictions of design codes are made.

\section{DESIGN OVERVIEW}

The SPARC project [1], which is an INFN-ENEAuniversity collaboration centered at the INFN-LNF (Frascati) lab, is designed to demonstrate a state-of-theart, high brightness electron source, and utilize this source for experiments. These experiments demand the highest beam performance levels, and include radiation production (e.g. FEL [2] and inverse Compton scattering) and advanced acceleration schemes (e.g. laser-plasma and inverse FEL) [3].

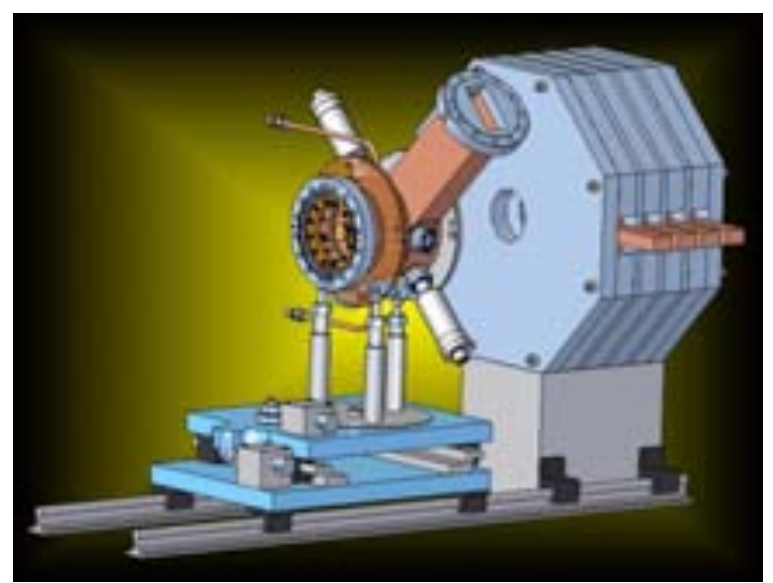

Figure 1. Schematic of SPARC photoinjector RF gun and emittance compensation solenoid. Supports not as built, cathode flange removed.

The electron source for SPARC, shown schematically in Fig. 1, is very close in design to the photoinjector sources designed for the LCLS X-ray SASE FEL [4], and deployed recently at the NLCTA-ORION. The source is based on the 1.6 cell $2856 \mathrm{MHz}$ BNL/SLAC/UCLA RF photoinjector gun, run at high gradient (120-140 MV/m on-axis field), which demands 11-15 MW of RF power. It is fitted with $\mathrm{Cu}$ or higher-quantum efficiency $\mathrm{Mg}$ photocathodes. The cathode is illuminated with a $266 \mathrm{~nm}$ pulse of frequency-tripled laser light which is spatially and temporally shaped into a flat-top, uniform intensity profile [2].

The gun is followed by an emittance compensation solenoid approximately $20 \mathrm{~cm}$ in length, and a drift to a linac section (entrance $150 \mathrm{~cm}$ from the cathode). This linac section has an additional solenoid focusing applied around it, to aid in the emittance compensation process, as called for in the so-called Ferrario working point [4] that forms the basis of many high brightness designs at present.

Both the gun and emittance compensation solenoid were redesigned through collaborative work at UCLA and INFN, with fabrication and initial testing occurring at UCLA. The 1.6 cell gun is close in design to the ORION model [5], which was also created by UCLA in collaboration with SLAC. Recent lessons learned from the ORION gun include, most importantly, the need to remove the insertable (at the outer wall) tuners from the full cell. This avoids breakdown at modest levels of RF power in the gun that afflicts nearly all known version of this gun. The ORION gun [5], with its tuners essentially out of the circuit, has been conditioned to over $15 \mathrm{MW}$ with little arcing; similar experience is reported at the BNL ATF [6].

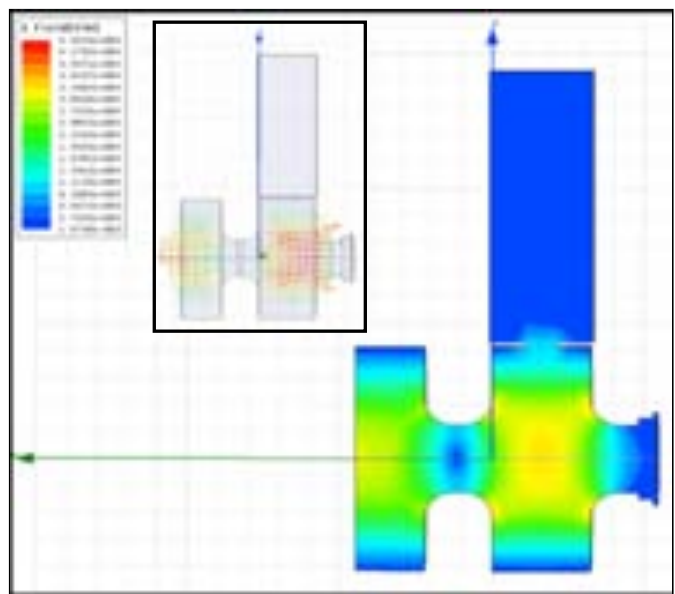

Figure 2. Output of HFSS, showing coupling to waveguide, and field balance in gun.

Thus a major goal of the SPARC gun construction is to remove the reliance on full cell tuners, which also serves to remove much of the quadrupole field component of the 
RF profile. It will be seen that removal of the tuners yields gun improvement in the general RF behavior.

In the course of the design process, the gun was simulated using 2- and 3-D codes. In order to model the coupling of the gun to the wave-guide, as well as to accurately predict power dissipation at this relatively high average power $(10 \mathrm{~Hz}, 3 \mu \mathrm{sec}$ RF fill $)$ HFSS 3D calculations were performed, as illustrated in Fig. 2.

The SPARC solenoid was designed to be similar to, but more flexible than, the standard BNL/LCLS type of device. Like previous versions, it employs field stiffening iron between coil sections, but in our case there are only four sections (see Fig. 1). Further, these coils are independently powered, in order to tailor the field profile. By asymmetrically exciting the coils (Fig. 3), this centroid of the field can be made with variable center in $z$, thus allowing choice of distance from the cathode to the effective solenoid lens center. The effect of this variation on the emittance compensation process is shown in Fig. 4. This "knob" will be used in the first phase of the project (no linac post-acceleration) to experimentally identify the optimum conditions for emittance compensation.

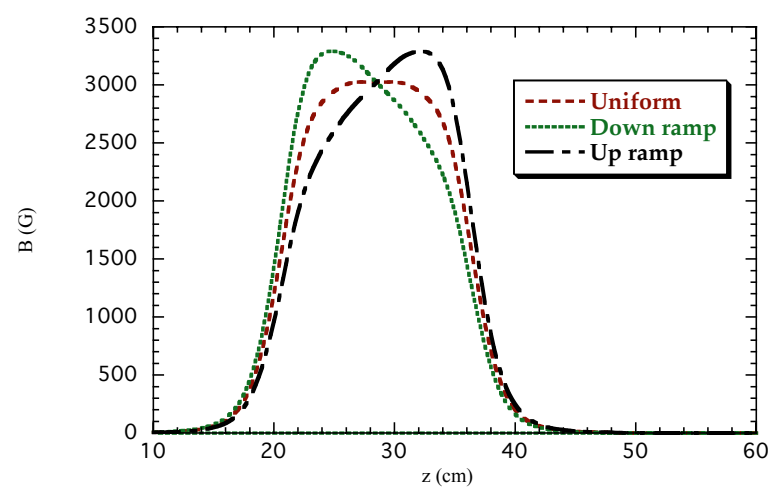

Figure 3. Field profiles in flat, ramp-up and ramp-down excitations of the SPARC solenoid, $60 \%$ variation of current between end coils.

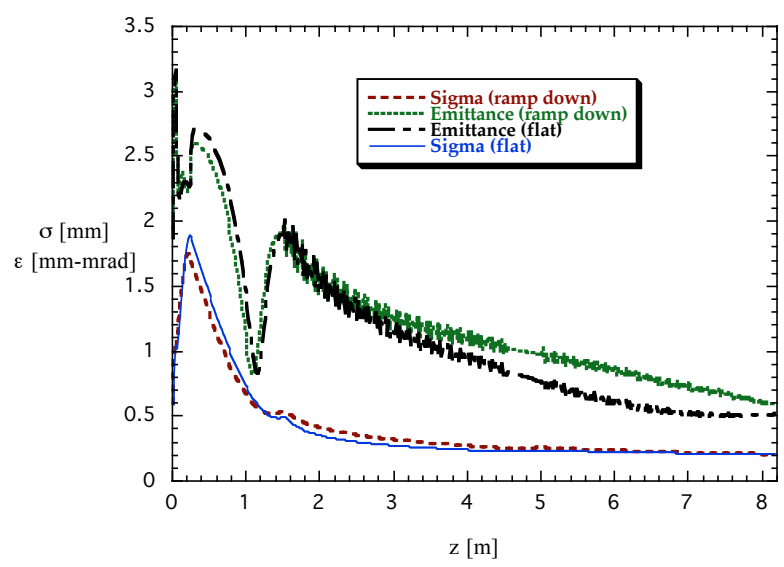

Figure 4. Rms beam size and emittance evolution in the SPARC injector, comparison between flat and ramp-down solenoid settings.
The solenoid was simulated using both POISSON (2D approx.) and Radia (3D). It was constructed with an octagonal outer yoke shape, and utilized coils that have large reservoir cooling, instead of center-core cooling. This allowed a current of 300 A (total current per coil section of 21,600 A-turns).

\section{RF GUN PERFORMANCE}

After fabrication and tuning at UCLA and SLAC, the gun underwent final testing at UCLA, and was shipped to Frascati in Jan. 2005. The testing involved: the identification of 0 and $\pi$-mode frequencies and their separation; the $\pi$-mode field balance, quality factor $Q$; external coupling $\beta$; and full cell coupling loop calibration. The results of these measurements are summarized in Table 1.

All measurements require that the gun field be balanced to yield correct running conditions. This balancing is accomplished by deforming the cathode shape using a tuning nut on the exterior center of the cathode. The (excellent) results of this optimization are shown in Figure 5 .

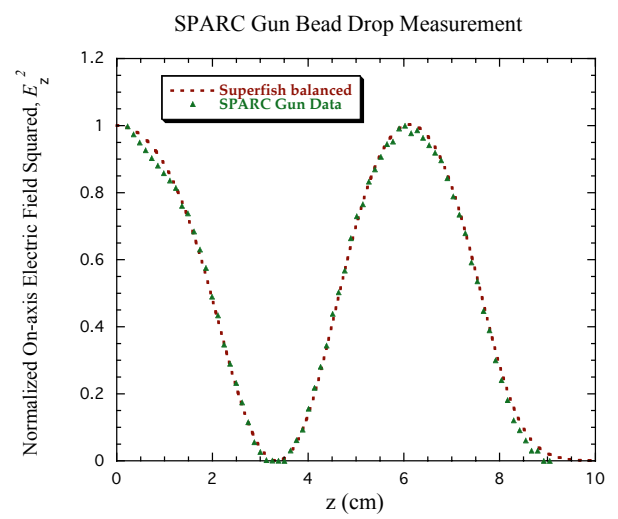

Figure 5. Bead drop data analyzed to show $E_{z}^{2}$, along with SUPERFISH prediction. Deviations near $z=0$ are caused by image charge effects.

Table 1. SPARC gun measurement summary.

\begin{tabular}{|l|l|}
\hline Mode separation $(0-\pi)$ & $3.06 \mathrm{MHz}$ \\
\hline Coupling $\beta$ (also VSWR) & 1.22 \\
\hline$Q_{0}$ & 12,500 \\
\hline Reflected power & $1 \%$ \\
\hline Running temperature & $42^{\circ} \mathrm{C}$ \\
\hline Full cell coupling calib. & $-65.4 \mathrm{~dB}$ \\
\hline
\end{tabular}

Despite the use of a standard power slot, the external coupling was found to be somewhat higher than in the past $(\beta=1.22)$, or $1 \%$ reflected power. This small reflection has no significance for operations, but does indicate an interesting difference from past devices - the 
cavity $Q_{0}$ was found to be 12,500 , which is roughly $8 \%$ higher than in past guns, and (now) in agreement with simulations. This implies that significant power loss was occurring in the tuner joints.

The full-cell power monitor coupling loop was calibrated, but it was noted that this measure-ment was quite sensitive to temperature, as the differential expansion of steel (the coupler tube) and copper changes the position of the loop in the evanescent field region. Final calibration must be performed at the operating temperature.

\section{SOLENOID PERFORMANCE}

The solenoid $B_{z}$ profile was scanned using Hall probes, for two different scenarios: $150 \mathrm{~A}$ in all for coils, and 150 A in only two downstream coils. The results are compared to the predictions of Radia (Fig. 6) 3D and Poisson (Fig. 7) $2 \mathrm{D}$ simulations. The overall profile is accurate in both cases, but the 2D model overestimates the field amplitude by $\sim 4 \%$. This discrepancy is in part due to the yoke geometry being octagonal, not cylindrical, as assumed in the 2D model.

The case of Fig. 6 corresponds to the setting required for running the gun at $132 \mathrm{MV} / \mathrm{m}$. As the coils can be run at nearly twice this current, a considerable overhead is available in $B_{z}$. This overhead will allow maximum flexibility in the differential excitation in the four coils.

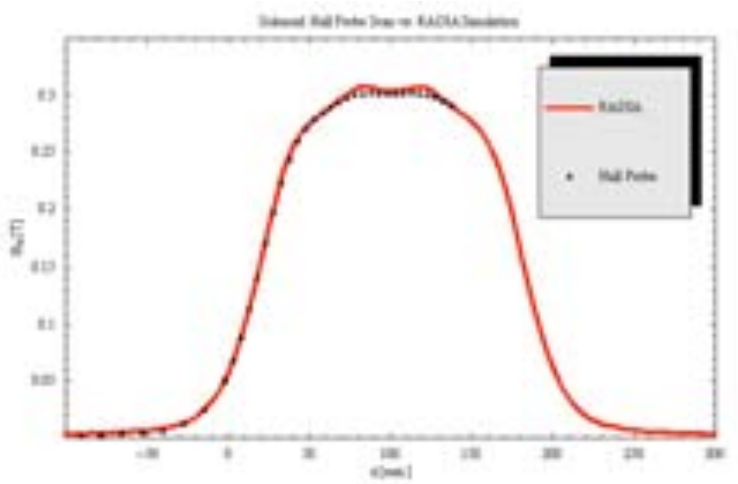

Figure 6. SPARC solenoid Hall probe scan (150 A on all coils); predictions of Radia 3D model.

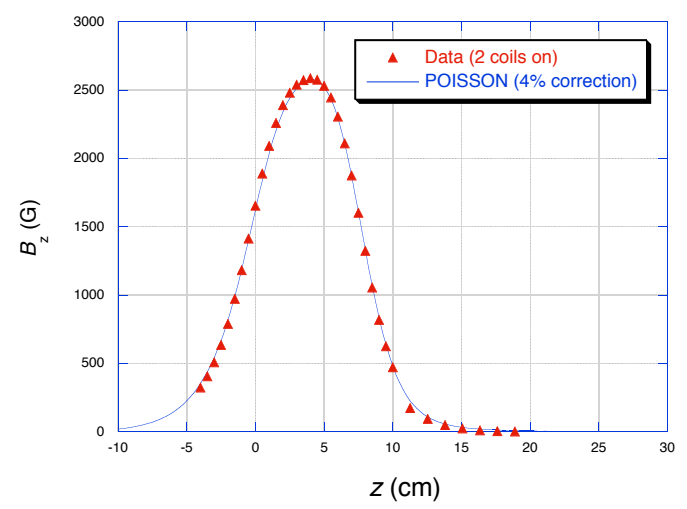

Figure 7. SPARC solenoid Hall probe scan with only two downstream coils excited at $150 \mathrm{~A}$; predictions of POISSON model ( $4 \%$ correction).
Perhaps the most important measurement to check in the scan of Fig. 6, however, is the residual field at the cathode position. For the measured conditions, this residual field is $7 \mathrm{G}$. This corresponds, for a $1 \mathrm{~mm}$ radius (design) beam spot, to a contribution of $0.03 \mathrm{~mm}-\mathrm{mrad}$ to the normalized emittance, an ignorable value.

The magnetic center of the solenoid was determined using a pulsed-wire apparatus. It was observed that the magnetic center was aligned to the mechanical center of the solenoid, to better than two wire widths (100 microns), when the adjustment screws were positioned all the way out (coils resting directly in the yoke support).

\section{OUTLOOK}

The SPARC photoinjector gun is due to be installed and aligned at the SPARC facility the LNF in June of 2005 , with first high power RF conditioning in October 2005. At that point, dedicated measurements of the RF and magnetic performance of the system will begin, commencing with the measurement of emittance evolution at low energy (5.5 MeV) as a function of laser, RF gun, and magnet parameters. The emittance will be characterized also as a function of distance from the gun. During this phase of experiments, it is also expected that different cathodes may be studied.

After this initial period of testing, the full acceleration system will be installed. This will allow the beam energy to be increased to above $150 \mathrm{MeV}$, and allow the full range of beam dynamics experiments (e.g. velocity bunching, RF deflector-based diagnostics), and FEL studies to begin.

\section{REFERENCES}

[1] D. Alesini et al., NIM A 528 (2004) 586.

M. Ferrario et al., Proc. EPAC-2004, pag. 399, ISBN: 92-9083-231-2A

Renieri et al., Status report on the SPARC Project, Proc. of FEL-2004.

[2] Technical Design Report for the SPARC Advanced Photoinjector, Eds. L. Palumbo and J. Rosenzweig, www.lnf.infn.it .

[3] D. Alesini et al., The Project PLASMONX for Plasma Acceleration Experiments and a Thomson X-ray Source at SPARC, these proceedings.

[3] M. Ferrario et al., Homdyn Studies for the LCLS RF Photoinjector, in The Physics of High Brightness Beams, J.Rosenzweig and L.Serafini ed., World Sci. ISBN 981-02-4422-3, June 2000

[5] D.T. Palmer, et al., The ORION Photo-injector, these proceedings.

[6] V. Yakimenko, private communication. 